\title{
Development of Deletion Lines for Chromosome 3D of Bread Wheat
}

\section{OPEN ACCESS}

Edited by:

Luigi Cattivelli,

Council for Agricultural and

Economics Research, Italy

Reviewed by:

Andrea Brandolini,

Council for Agricultural and

Economics Research, Italy

Adam Lukaszewski,

University of California, Riverside,

United States

*Correspondence:

Jan Bartoš

bartos@ueb.cas.cz

Specialty section:

This article was submitted to

Plant Breeding,

a section of the journal

Frontiers in Plant Science

Received: 25 October 2019 Accepted: 16 December 2019

Published: 28 January 2020

Citation:

Svačina $R$, Karafiátová $M$, Malurová $M$, Serra $H$, Vitek $D$, Endo TR, Sourdille $P$ and Bartoš J (2020) Development of Deletion Lines for Chromosome 3D of Bread Wheat.

Front. Plant Sci. 10:1756. doi: $10.3389 /$ fp/s.2019.01756

\author{
Radim Svačina ${ }^{1}$, Miroslava Karafiátová ${ }^{1}$, Magdaléna Malurová $^{1}$, Heïdi Serra ${ }^{2}$, \\ Dominik Vitek ${ }^{1}$, Takashi R. Endo ${ }^{3}$, Pierre Sourdille ${ }^{2}$ and Jan Bartos ${ }^{1 *}$ \\ 1 Institute of Experimental Botany, Czech Academy of Sciences, Centre of the Region Hana for Biotechnological and \\ Agricultural Research, Olomouc, Czechia, ${ }^{2}$ INRA, Génétique, Diversité, Ecophysiologie des Céréales, Clermont-Ferrand, \\ France, ${ }^{3}$ Faculty of Agriculture, Ryukoku University, Shiga, Japan
}

The identification of genes of agronomic interest in bread wheat (Triticum aestivum L.) is hampered by its allopolyploid nature $(2 n=6 x=42$; AABBDD) and its very large genome, which is largely covered by transposable elements. However, owing to this complex structure, aneuploid stocks can be developed in which fragments or entire chromosomes are missing, sometimes resulting in visible phenotypes that help in the cloning of affected genes. In this study, the 2C gametocidal chromosome from Aegilops cylindrica was used to develop a set of 113 deletion lines for chromosome 3D in the reference cultivar Chinese Spring. Eighty-four markers were used to show that the deletions evenly covered chromosome 3D and ranged from 6.5 to $357 \mathrm{Mb}$. Cytogenetic analyses confirmed that the physical size of the deletions correlated well with the known molecular size deduced from the reference sequence. This new genetic stock will be useful for positional cloning of genes on chromosome 3D, especially for Ph2 affecting homoeologous pairing in bread wheat.

Keywords: wheat, deletion line, homoeologous pairing, Ph2, gametocidal

\section{INTRODUCTION}

Bread wheat (Triticum aestivum L.) is one of the most important cultivated crops. It emerged through two distinct hybridization events between three diploid species, resulting in its allohexaploid nature. The genetic material consists of three closely related subgenomes, namely A, B, and D (Huang et al., 2002), which generate the genomic plasticity necessary for bread wheat to grow under a wide range of climatic conditions. Moreover, bread wheat tolerates the creation of aneuploid lines, such as nullisomic, substitution, deletion, and many other types. However, the three sets of homoeologous chromosomes create a vulnerability to incorrect chromosome pairing during meiosis, possibly resulting in aberrant gametes. Therefore, to maintain the pairing behavior during meiosis, a system developed in wheat that is enforced genetically by pairing homoeologues $(P h)$ genes. In this control, the most effective genes are $P h 1$ and $P h 2 . P h 1$ is on the 5B chromosome and has a major influence on homoeologous chromosome pairing (Riley and Chapman, 1958; Sears and Okamoto, 1958). Ph2 is on the short arm of chromosome 3D (Mello-Sampayo, 1971) and has less of an effect compared with Ph1. Despite some attempts at positional cloning (Sutton et al., 2003), Ph2 has not been formally identified to date. Other genes contribute to the control of homoeologous pairing but have only minor influence, such as $P h 3$, which is on the short arm of chromosome $3 \mathrm{~A}$ and is possibly a homoeologous variant of Ph2 (Driscoll, 1972; Mello-Sampayo and Canas, 1973). 
Genes are usually maintained in a population by benefiting their hosts or alternatively, by high linkage to such a gene (a phenomenon called linkage-drag). However, there are exceptions to this rule, such as transposable elements, B chromosomes, and gametocidal genes/chromosomes. These genetic units use various "selfish" behaviors to either preserve their existence in the population or to increase their number. The gametocidal genes or chromosomes secure their inheritance to progeny through induction of genomic aberrations and consequent total or partial sterility in gametes lacking them. In wheat, this phenomenon is observed in substitution and addition lines with alien chromosomes from the genus Aegilops. The backcrossing of hybrids to wheat between the two species does not remove certain chromosomes of Aegilops from the genome of progeny (Endo and Tsunewaki, 1975; Maan, 1975), and chromosomal aberrations are observed in some gametes of such hybrids (Finch et al., 1984). Gametocidal chromosomes originate from the Aegilops genomes $\mathrm{C}, \mathrm{S}$, and $\mathrm{M}$, and the magnitude of their effect in wheat varies with the type of gametocidal chromosome and the genotype of the wheat background. Whereas some chromosomes cause complete sterility of gametes that lack them (e.g., $2 S^{\text {lo }}, 2 S^{\text {sh }}$, T2B- $2 S^{\text {sp.au }}, 4 S^{\text {lo }}, 4 S^{\text {sh }}$, and $4 S^{\text {sh }} \# 2$ ); others generate only semi-lethal changes and make it possible to transfer the aberrations to progeny (Endo, 1990; Endo, 2007).

The 2C gametocidal chromosome from Aegilops cylindrica has been introduced to the T. aestivum 'Chinese Spring' background and is being exploited to create mostly terminal deletions of wheat chromosomes. Hereafter, this procedure will be called the "2C gametocidal system" (Endo and Gill, 1996). Tsujimoto (1993) showed that telomeric regions are quickly rebuilt after chromosome breakage and that chromosome stability allows this system to be used as a genetic tool. Endo and Gill (1996) produced 436 deletion lines across all chromosomes using this approach, with subsequent establishment of deletion chromosomes in homozygous/ hemizygous constitutions. This resource has been a powerful tool in mapping the position of various genes and markers (Sourdille et al., 2004).

The $2 \mathrm{C}$ gametocidal system can be used to create a series of aberrations in any chromosome of wheat. However, the judicious use of existing aneuploid stocks can increase the efficiency and ease in selecting aberrations targeting specific chromosomes. If the monosomic addition line $2 \mathrm{C}$ is crossed as male to a nulli-tetrasomic line lacking the targeted chromosome, the recovered aberrations will be monosomic and hence easily detectable by PCR-based techniques. The selection of disomic/ homozygous aberrations is performed following self-pollination of the plants carrying the aberrations, but the presence of the additional copy of a homoeologue inherited from the nullitetrasomic may complicate the transmission patterns of the targeted chromosome.

In this study, the $2 \mathrm{C}$ gametocidal system was used to develop a set of deletion lines for chromosome 3D in wheat to map the position of the $P h 2$ gene. This gene was previously mapped using a ph 2 a mutant carrying a terminal deletion on chromosome $3 \mathrm{D}$ that was estimated to be approximately $80 \mathrm{Mb}$ (Sutton et al., 2003).

\section{MATERIALS AND METHODS}

\section{Plant Material and Crosses}

The deletion lines were derived from crosses between the monosomic addition line of chromosome $2 \mathrm{C}$ from A. cylindrica in the hexaploid wheat cultivar Chinese Spring (CS) background $\left(6 \mathrm{x}=2 \mathrm{n}=43\right.$; AABBDD $\left.+2 \mathrm{C}^{\prime}\right)$ used as male and the hexaploid $\mathrm{CS}$ wheat nulli-tetrasomic lines lacking chromosome $3 \mathrm{D}$ with tetrasomic constitution either for chromosome $3 \mathrm{~A}$ or $3 \mathrm{~B}(6 \mathrm{x}=$ $2 \mathrm{n}=42$; AABBDD $-3 \mathrm{D}^{\prime \prime}+3 \mathrm{~A}^{\prime \prime} / 3 \mathrm{~B}^{\prime \prime}$ ) used as female (Figure 1). The 2C gametocidal chromosome induces chromosomal breakages in gametes where it is not transferred, resulting

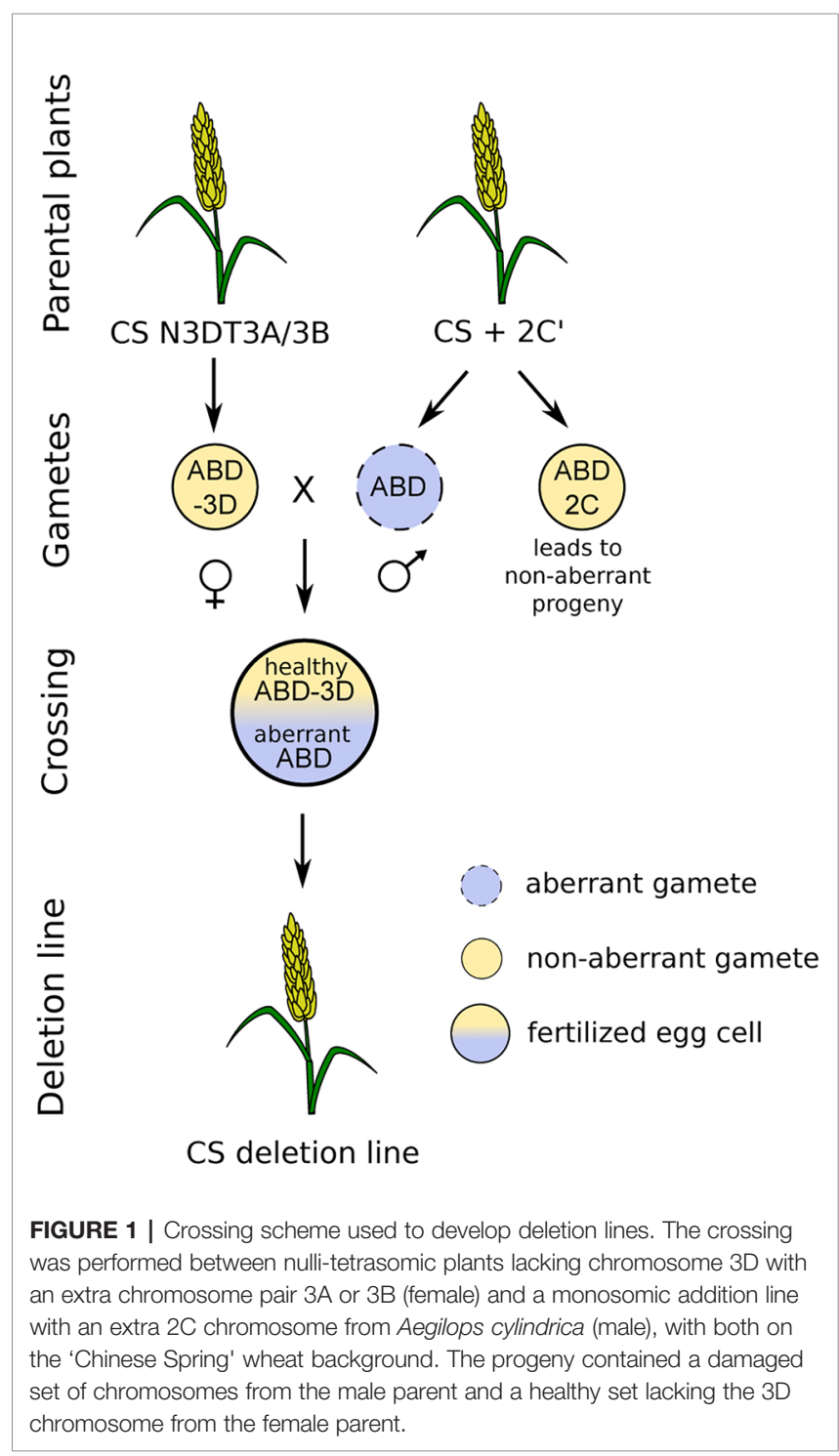


mostly in terminal deletions. The crosses with nulli-tetrasomic lines lacking a pair of $3 \mathrm{D}$ chromosomes ensure that a potentially aberrant $3 \mathrm{D}$ chromosome from the $2 \mathrm{C}$ addition-line parent will be in the progeny in a monosomic state and that a deletion will not be masked by an entire 3D chromosome from the female parent. The plants were cultivated in growth chambers under the following conditions: a $16 / 8 \mathrm{~h}$ light/dark photoperiod, temperatures of $20^{\circ} \mathrm{C}$ during the day and $16{ }^{\circ} \mathrm{C}$ at night, and $60 \%$ humidity.

\section{Identification of Plants With Deletion on the 3D Chromosome}

The seeds acquired from the crosses were germinated in pots and cultivated for 2 weeks. Thereafter, DNA was isolated from a part of a young leaf by using a magnetic beads protocol (Sbeadx mini plant kit, LGC, Teddington, United Kingdom). The DNA was used to identify the deletion lines in the F1 generation. Molecular markers were designed for the distal ends of both arms of chromosome $3 \mathrm{D}$, with a marker located in the centromeric area as a control for chromosome presence; primer details are shown in Table 1. The PCR was performed in $20 \mu \mathrm{l}(1 \times \mathrm{PCR}$ buffer, $1.5 \mathrm{mM} \mathrm{MgCl}, 200 \mu \mathrm{M}$ dNTPs, $1 \mu \mathrm{M}$ primers, $20 \mathrm{ng}$ of DNA, $0.4 \mathrm{U} / 20 \mu \mathrm{l}$ Taq DNA polymerase) under the following conditions: initial denaturation at $95^{\circ} \mathrm{C}$ for $10 \mathrm{~min}$; 35 cycles of denaturation at $95{ }^{\circ} \mathrm{C}$ for $30 \mathrm{~s}$, annealing at $60{ }^{\circ} \mathrm{C}$ for $30 \mathrm{~s}$, and elongation at $72{ }^{\circ} \mathrm{C}$ for $50 \mathrm{~s}$; followed by a final extension at $72{ }^{\circ} \mathrm{C}$ for $10 \mathrm{~min}$. The PCR was scored for the presence/absence of a specific product on $1.2 \%$ agarose gel. The plants carrying a deletion on chromosome 3D (lacking either or both 3DS and 3DL-specific PCR products) were replanted into larger pots and cultivated under the following conditions: a $16 / 8 \mathrm{~h}$ light/dark photoperiod, temperatures of $20^{\circ} \mathrm{C}$ during the day and $16{ }^{\circ} \mathrm{C}$ at night, and $60 \%$ humidity. Plants were grown until seed harvest.

\section{Characterization of Sizes of Deletions}

The deletion lines of chromosome 3D were characterized using a set of STS molecular markers covering the entire chromosome. In addition to the deletion lines, the X-ray-induced deletion mutant ph2a (Sears, 1950) was also characterized. Eighty-four was the final number of markers (Supplementary Table 1), of which 58 were on the short arm and 26 were on the long arm of the chromosome. The characterization was performed using presence/absence scoring and agarose gel electrophoresis separation as described above.

The primers for analysis were designed using the reference sequence of the wheat genome (IWGSC, 2018). The sequence was masked for annotated repetitive sequences. The loci for

TABLE 1 | Sequences and localization of primers used for identification of lines carrying a deletion on the short arm, long arm, or both arms of chromosome 3D.

\begin{tabular}{lll}
\hline Oligo ID & Sequence 5'-3' & Localization \\
\hline 3D_0.3Mb_F & TTAGTGGATCGAGGATTGTG & distal 3DS \\
3D_0.3Mb_R & TCGGTGACTAGTGTGTTCTG & \\
3D_610.2Mb_F & GCAACAGAAGAAGAAATACTGCT & distal 3DL \\
3D_610.2Mb_R & GTGCATCATATCTATGGTCTATC & \\
3D_253.4Mb_F & TATGCGTTTGGAGTAGTTCTTGT & 3D centromere \\
3D_253.4Mb_R & CTCATCTCAGGCTGTCTAATTAA &
\end{tabular}

primer design were selected to cover the chromosome as evenly as possible with the priority in the distal $125 \mathrm{Mb}$ of the short arm of chromosome 3D. The regions not masked with repeats (10-30 $\mathrm{kb}$ ) were aligned using BLASTn against reference sequences of chromosomes $3 \mathrm{~A}$ and $3 \mathrm{~B}$, and the corresponding regions were compared to depict the $3 \mathrm{D}$-specific polymorphisms. Those polymorphisms were used to design $3 \mathrm{D}$-specific primers (Supplementary Table 1). The primers were tested on $T$. aestivum 'Chinese Spring' as the positive control, a nullitetrasomic line lacking chromosome $3 \mathrm{D}$ as the negative control, and water as the blank.

\section{Identification of Deletion Lines With the 3D Chromosome in a Disomic State}

Each deletion line was self-pollinated to increase seed stocks and to induce a disomic constitution of the $3 \mathrm{D}$ chromosome carrying a deletion. The upcoming generation comprised nullisomics, monosomics, and disomics for the analyzed chromosome. Therefore, screening with molecular markers was necessary to select the stable lines carrying the 3D chromosome with deletion in disomic constitution. First, the entire population was screened using the PCR marker on the centromere of the $3 \mathrm{D}$ chromosome (see above) to eliminate all nullisomics. The plants carrying the 3D chromosome were selected for droplet digital PCR (ddPCR) analysis. The ddPCR analysis was performed using ddPCR ${ }^{\mathrm{TM}}$ Supermix for Probes (no dUTP) (Bio-Rad, Hercules, USA) according to manufacturer's instructions with a $60{ }^{\circ} \mathrm{C}$ annealing/extension phase. The reference and target primers and TaqMan ${ }^{\circledR}$ probes (Thermo Fisher Scientific, Waltham, USA) used for chromosomes 4A (disomic in all lines) and 3D are listed in the Table 2.

\section{Fluorescent in Situ Hybridization of Selected Lines}

Selected lines carrying a deletion on the $3 \mathrm{D}$ chromosome were characterized cytogenetically using fluorescent in situ hybridization (FISH). Mitotic metaphase chromosomes were obtained from synchronized root tip meristems (Vrána et al., 2012). Synchronized roots were fixed in $90 \%$ ice-cold acetic acid for $10 \mathrm{~min}$ and then washed three times with $70 \%$ ethanol and stored at $-20{ }^{\circ} \mathrm{C}$ in $70 \%$ ethanol. Chromosome preparations using the drop technique were performed according to Danilova et al. (2012). The individual chromosomes in the wheat karyotype were identified using the combination of two FISH probes: $(\mathrm{GAA})_{\mathrm{n}}$ microsatellite (FITC) and Afa repeat (Cy3) (Pedersen and Langridge, 1997; see Supplementary Figure 1). The probes were labeled via PCR, and FISH was performed under the conditions described in Kubaláková et al. (2003). The signals were observed using a Zeiss Axio Imager Z2 fluorescent microscope (Carl Zeiss, Jena, Germany) equipped with a CCD camera. At least five copies of the 3D chromosome per line were characterized by measurement of the deleted arm and whole chromosome length by using MicroImage software version 4.0 (Olympus, Shinjuku, Japan). The deletion size on chromosome $3 \mathrm{D}$ was estimated on the basis of the fragment length value (Endo and Gill, 1996). 


\section{RESULTS}

From the F1 generation, 6169 seeds formed by crosses between the monosomic addition line of chromosome $2 \mathrm{C}$ from $A$. cylindrica and the nulli-tetrasomic lines $(6 \mathrm{x}=2 \mathrm{n}=42$; AABBDD - 3D" $\left.+3 \mathrm{~A}^{\prime \prime} / 3 \mathrm{~B}^{\prime \prime}\right)$ were analyzed. The plants carrying a deletion on chromosome $3 \mathrm{D}$ were detected using STS markers designed for the terminal ends of 3D chromosomal arms. In total, 113 deletion lines were developed (Supplementary Table 2). All identified plants in the F1 generation carried a $3 \mathrm{D}$ chromosome with a deletion in monosomic constitution. More precisely, 43 (39.13\%) of the lines carried a deletion on 3DS, $68(60.87 \%)$ carried a deletion on $3 \mathrm{DL}$, and two lines carried a deletion on both arms (for the schematic layout, see Supplementary Figures 2, 3, and 4). These numbers corresponded to the length-arm ratio of 0.393 $(240 \mathrm{Mb})$ for the short arm and $0.607(370 \mathrm{Mb})$ for the long arm (IWGSC, 2018).

A set of self-pollinations established a disomic constitution of deleted chromosomes in individual lines. The number of 3D copies was analyzed using a ddPCR protocol with specific primers and the TaqMan probe system comparing the number of events on the analyzed chromosome (3D) with that on the reference chromosome (4A). The disomic constitution of deletion chromosomes was successfully established in 102 of the 113 lines.

The whole set of deletion lines was characterized using 84 STS molecular markers evenly distributed along the entire 3D chromosome (Supplementary Table 1). The size of the deletions ranged from 6.5 to $357 \mathrm{Mb}$, and the size of the deletion bins (the region between two adjacent deletion breakpoints) ranged from 0.15 to $50 \mathrm{Mb}$. Some deletions seemed to have the same breakpoint; however, this was most likely caused by insufficient resolution of molecular markers in that particular region. The length of chromosome arm deletions and the number of missing genes in individual lines, as well as the differences in missing genes among the lines, are summarized in Supplementary Table 2.

The deletion lines of chromosome 3D were produced to map the position of the $\mathrm{Ph} 2$ gene that was localized on this chromosome by Mello-Sampayo (1971). The position of this gene was further delimited using an X-ray-induced deletion

TABLE 2 | Sequences of primers and probes used for determination of 3D chromosome number in the ddPCR assay. The TaqMan (taq) probes were either labelled by FAM (4A chromosome; used as a reference) or VIC (3D chromosome; target).

\begin{tabular}{llc}
\hline Oligo ID & Sequence and modifications 5'-3' & $\begin{array}{c}\text { Amplicon } \\
\text { length [bp] }\end{array}$ \\
\hline Ta-4A_F & ATTTGGGTCCTTGTTGTATC & 181 \\
Ta-4A_R & ACACGCATGAAGTGTATAATGC & \\
Ta-4A_taq & FAM-AAGAACTTCACACACGAACTCGGA-QSY & \\
Ta-3D_F & CTCATCTCAGGCTGTCTAATTAA & 167 \\
Ta-3D_R & CATAGATCCCTCCTTGAAGGA & \\
Ta-3D_taq & VIC-CCTCACTCAAGCACCACATCG-QSY &
\end{tabular}

mutant ph2a (Sears, 1982), and therefore, this mutant was included in the analysis as a control. The size of the deletion in the ph 2 a mutant was previously estimated to affect approximately $80 \mathrm{Mb}$ in the terminal part of the 3DS using synteny with the rice chromosome (Sutton et al., 2003). However, the screening by molecular markers showed this deletion to be larger by approximately $40 \mathrm{Mb}$, because the breakage point was between 120 and $125 \mathrm{Mb}$.

FISH analysis of selected deletion lines representing various lengths of deletions was performed to cytogenetically characterize the material (Figure 2). The 3D chromosome was identified using the Afa repeat family (Pedersen and Langridge, 1997). Among the 32 selected deletion lines, 12 had the breakage on the 3DS and 20 had the breakage on the 3DL. In all cases, the size of deletion determined by molecular markers was confirmed by cytogenetic observation.

\section{DISCUSSION}

The deletion lines were produced using the gametocidal system described by Endo and Gill (1996). The 2C gametocidal chromosome causes terminal chromosomal deletions in the gametes that lack it. However, these aberrations are usually not lethal because of the compensation by the other two homoeologous chromosomes. Thus, deletions can be transferred into progeny (Endo, 1988). Endo and Gill (1996) derived 436 plants via this system and characterized the deletions cytogenetically using a C-banding protocol. Of the 436 plants, 12 of them carried a deletion on chromosome 3D. In this study, 113 novel deletion lines for chromosome 3D were generated, increasing substantially the number of chromosome $3 \mathrm{D}$ deletion lines that are available for use in various applications.

Because the deletion lines were primarily produced to map the $P h 2$ gene, the marker resolution was highest on the short arm of chromosome 3D. The 58 markers divided the short arm into segments ranging from $100 \mathrm{~kb}$ to a maximum of $29 \mathrm{Mb}$ in the centromeric area. Owing to the high marker resolution, only a single or a few deletion breakpoints were assigned in each segment (Supplementray Table 2). The short arm of chromosome 3D comprises 1,949 annotated genes (IWGSC, 2018), and in this study, the estimated number of genes deleted in individual lines was 194-1,927, with the number of genes in each deletion bin ranging from 7 to 276 . By contrast, the resolution achieved with 26 markers on the long arm of the chromosome was lower than that on the short arm, with segments ranging from 3 to $50 \mathrm{Mb}$. The long arm of chromosome 3D carries 3,369 genes (IWGSC, 2018), and the number of genes deleted in individual lines ranged from 306 to 3,351, with each deletion bin comprising between 76 and 468 genes (see Supplementray Table 2). The resolution of deletion bins in the area of the Ph2 gene (distal $125 \mathrm{Mb}$ of the short arm) ranged between 1.5 and $12 \mathrm{Mb}$, with an average of $6 \mathrm{Mb}$, limiting the number of potential candidate genes to between 7 and 276 .

To produce single chromosome deletion lines via the $2 \mathrm{C}$ gametocidal system, a cross is performed with one parent a nulli- 


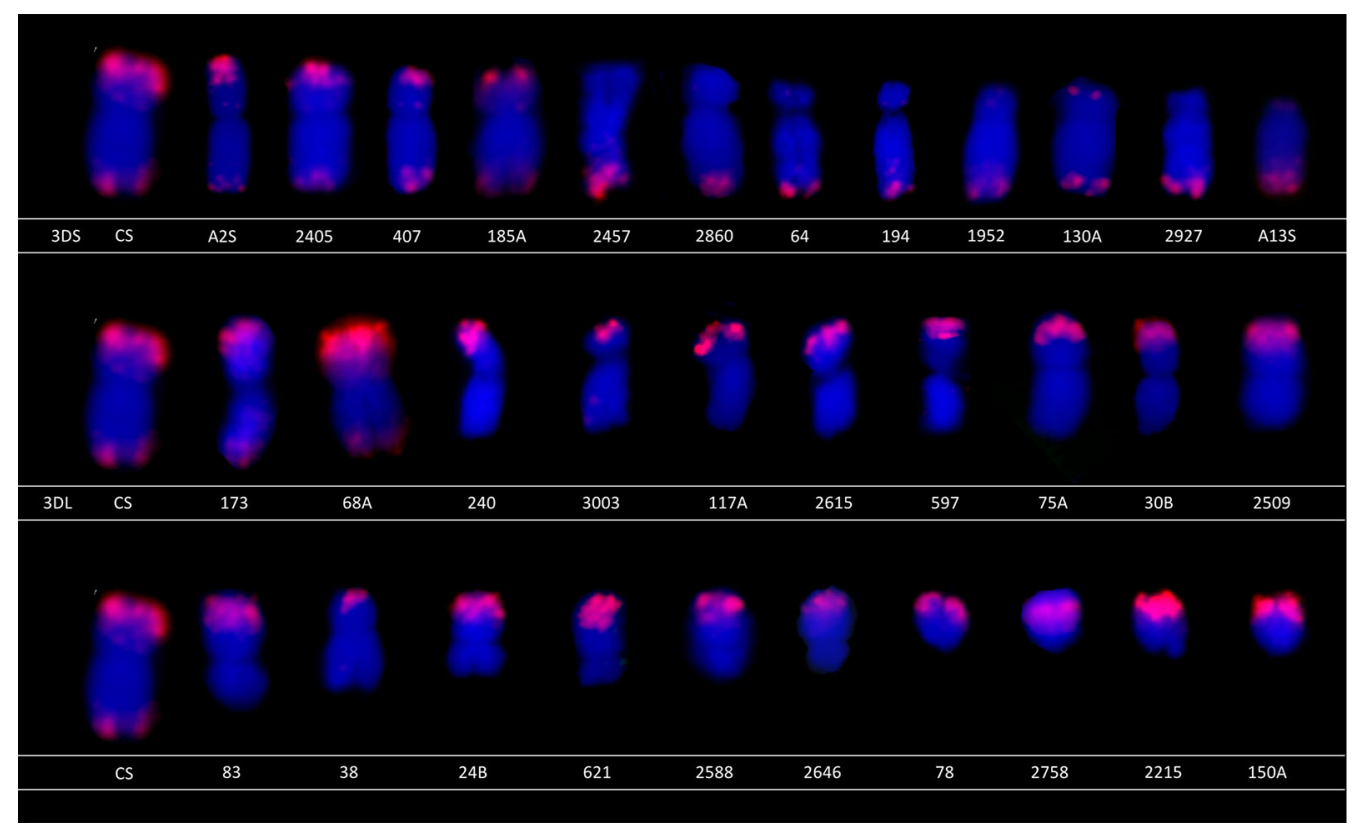

FIGURE 2 | Characterization of selected lines using fluorescence in situ hybridization (FISH). The selected lines were characterized using FISH to confirm the deletion size. The chromosomes were labeled using (GAA) microsatellite (green) and Afa repeat (red) to distinguish chromosome 3D from other chromosomes. Note that only Afa repeat is present on chromosome 3D.

tetrasomic line lacking a chromosome of interest. The resulting progeny carry the deleted chromosome of interest in a monosomic constitution. Because the gametes produced by the progeny may or may not contain the deleted chromosome, the lines are unstable for direct use, making it unreliable material for seed stock enlargement, crossing, or physical gene mapping. Therefore, self-pollination of this material is recommended to accumulate the deleted chromosome in a disomic constitution. The self-pollination of a plant carrying a chromosome in the monosomic state can produce nullisomic, monosomic, or disomic progeny for the respective chromosome. However, the proportion of transmission to progeny of such a chromosome is shifted by various irregularities in univalent behavior in meiosis. In Nicotiana tabacum, the univalent elimination of different monosomic chromosomes occurs at the same frequency, fluctuating around $75 \%$ (Olmo, 1935). In wheat, however, the univalent elimination seems to have greater variability, depending on which chromosome is in a monosomic state (Morrison and Unrau, 1952; Tsunewaki and Heyne, 1960). In the material in this study, nullisomics occurred more frequently than expected in progeny of monosomic deletion lines. Univalent behavior during meiosis can explain the unexpected proportions of nullisomics, monosomics, and disomics in progeny. Because univalents lag behind the bivalents while being pulled to the poles at anaphase I, they are therefore excluded from newly formed nuclei and are preserved in the cytoplasm as micronuclei (Sears, 1950; Tsunewaki and Heyne, 1960).

In this study, the $2 \mathrm{C}$ gametocidal system was used to develop novel deletion lines for chromosome 3D in common wheat (Endo and Gill, 1996). The deleted chromosome was successfully fixed in disomic constitution in most of the material to ensure the stable inheritance of the chromosome of interest, which greatly improves further use of the deletion lines. The new material will be useful to clone genes of agronomic interest, such as $P h 2$, a gene involved in homoeologous pairing in bread wheat (Mello-Sampayo, 1971).

\section{DATA AVAILABILITY STATEMENT}

All datasets generated for this study are included in the article/ Supplementary Material.

\section{AUTHOR CONTRIBUTIONS}

TE, PS, and JB designed the study. RS and TE crossed plants. RS, HS, and DV performed PCRs and ddPCR screening. MK and MM characterized the deletion lines using FISH. RS, PS, and JB wrote the manuscript. All authors approved the manuscript.

\section{FUNDING}

This work was supported by the Czech Science Foundation (grant award 17-05341S) and the ERDF project "Plants as a 
tool for sustainable global development" (CZ.02.1.01/0.0/0.0/ 16_019/0000827). We acknowledge the Investment for the Future programme BREEDWHEAT (project ANR-10-BTBR03) funded by the French Government and managed by the Research National Agency (ANR) for providing markers.

\section{REFERENCES}

Danilova, T. V., Friebe, B., and Gill, B. S. (2012). Single-copy gene fluorescence in situ hybridization and genome analysis: Acc-2 loci mark evolutionary chromosomal rearrangements in wheat. Chromosoma 121, 597-611. doi: 10.1007/s00412-012-0384-7

Driscoll, C. J. (1972). Genetic suppression of homoeologous chromosome pairing in hexaploid wheat. Can. J. Genet. Cytol. 14, 1. doi: 10.1139/g72-004

Endo, T. R., and Gill, B. S. (1996). The deletion stocks of common wheat. J. Hered. 87, 295-307. doi: 10.1093/oxfordjournals.jhered.a023003

Endo, T. R., and Tsunewaki, K. (1975). Sterility of common wheat with Aegilops triuncialis cytoplasm. J. Hered. 66, 13-16. doi: 10.1093/oxfordjournals. jhered.a108562

Endo, T. R. (1988). Induction of chromosomal structural changes by a chromosome of Aegilops cylindrica L. @ in common wheat. J. Hered. 79, 366-370. doi: 10.1093/oxfordjournals.jhered.a110529

Endo, T. R. (1990). GC chromosomes and their induction of chromosome mutations in wheat. Jpn. J. Genet. 65, 135-152. doi: 10.1266/jjg.65.135

Endo, T. R. (2007). The gametocidal chromosome as a tool for chromosome manipulation in wheat. Chromosome Res. 15, 67-75. doi: 10.1007/s10577-0061100-3

Finch, R. A., Miller, T. E., and Bennett, M. D. (1984). “Cuckoo” Aegilops addition chromosome in wheat ensures its transmission by causing chromosome breaks in meiospores lacking it. Chromosoma 90, 84-88. doi: 10.1007/BF00352282

Huang, S., Sirikhachornkit, A., Su, X. J., Faris, J., Gill, B., Haselkorn, R., et al. (2002). Genes encoding plastid acetyl-CoA carboxylase and 3phosphoglycerate kinase of the Triticum/Aegilops complex and the evolutionary history of polyploid wheat. Proc. Natl. Acad. Sci. U. S. A. 99, 8133-8138. doi: 10.1073/pnas.072223799

IWGSC. (2018). Shifting the limits in wheat research and breeding using a fully annotated reference genome. Science 361:eaar7191, 661. doi: 10.1126/ science.aar7191

Kubaláková, M., Valárik, M., Bartoš, J., Vrána, J., Číhalíková, J., Molnár-Láng, M., et al. (2003). Analysis and sorting of rye (Secale cereale L.) chromosomes using flow cytometry. Genome 46, 893-905. doi: 10.1139/g03-054

Maan, S. S. (1975). Exclusive preferential transmission of an alien chromosome in common wheat. Crop Sci. 15, 287-292. doi: 10.2135/cropscil 1975 . 0011183X001500030002x

Mello-Sampayo, T., and Canas, A. P. (1973). "Suppression of meiotic chromosome pairing in common wheat," in Proceedings of the 4th international wheat genetics symposium. Eds. E. R. Sears and L. M. S. Sears (Columbia, MO, USA: Agric. Exp. Stn.), 703-713s.

Mello-Sampayo, T. (1971). Genetic regulation of meiotic chromosome pairing by chromosome-3D of Triticum aestivum. Nat. New. Biol. 230, 22. doi: 10.1038/ newbio230022a0

Morrison, J. W., and Unrau, J. (1952). Frequency of micronuclei in pollen quartets of common wheat monosomics. Can. J. Bot. 30, 371. doi: 10.1139/b52-029

\section{SUPPLEMENTARY MATERIAL}

The Supplementary Material for this article can be found online at: https://www.frontiersin.org/articles/10.3389/fpls.2019.01756/ full\#supplementary-material

Olmo, H. P. (1935). Genetical Studies of Monosomic Types of Nicotiana tabacum. Genet 20, 286-300.

Pedersen, C., and Langridge, P. (1997). Identification of the entire chromosome complement of bread wheat by two-colour FISH. Genome 40, 589-593. doi: 10.1139/g97-077

Riley, R., and Chapman, V. (1958). Genetic control of the cytologically diploid behaviour of hexaploid wheat. Nature 182, 713-715. doi: 10 . 1038/182713a0

Sears, E. R., and Okamoto, M. (1958). "Intergenomic chromosome relationships in hexaploid wheat," in Proceedings of the Xth International Congress of Genetics. Ed. J. W. Boyes (Toronto, Canada: University of Toronto Press), 258-259. doi: 10.1007/bf00325789

Sears, E. R. (1950). Misdivision of univalents in common wheat. Chromosoma 4, 535-550. doi: 10.1007/bf00325789

Sears, E. R. (1982). A wheat mutation conditioning an intermediate level of homoeologous chromosome pairing. Can. J. Genet. Cytol. 24, 715-719. doi: 10.1139/g82-076

Sourdille, P., Singh, S., Cadalen, T., Brown-Guedira, G. L., Gay, G., Qi, L., et al. (2004). Microsatellite-based deletion bin system for the establishment of genetic-physical map relationships in wheat (Triticum aestivum L.). Funct. Integr. Genomics 4, 12-25. doi: 10.1007/s10142-004-0106-1

Sutton, T., Whitford, R., Baumann, U., Dong, C. M., Able, J. A., and Langridge, P. (2003). The Ph2 pairing homoeologous locus of wheat (Triticum aestivum): identification of candidate meiotic genes using a comparative genetics approach. Plant J. 36, 443-456. doi: 10.1046/j.1365313X.2003.01891.x

Tsujimoto, H. (1993). Molecular cytological evidence for gradual telomere synthesis at the broken chromosome ends in wheat. J. Plant Res. 106, 239244. doi: 10.1007/BF02344591

Tsunewaki, K., and Heyne, E. G. (1960). The transmission of the monosomic condition in wheat: var. Chin. Spring. J. Hered. 51, 63-68. doi: 10.1093/ oxfordjournals.jhered.a106953

Vrána, J., Šimková, H., Kubaláková, M., Číhalíková, J., and Doležel, J. (2012). Flow cytometric chromosome sorting in plants: The next generation. Methods 57, 331-337. doi: 10.1016/j.ymeth.2012.03.006

Conflict of Interest: The authors declare that the research was conducted in the absence of any commercial or financial relationships that could be construed as a potential conflict of interest.

Copyright (C) 2020 Svačina, Karafiátová, Malurová, Serra, Vitek, Endo, Sourdille and Bartoš. This is an open-access article distributed under the terms of the Creative Commons Attribution License (CC BY). The use, distribution or reproduction in other forums is permitted, provided the original author(s) and the copyright owner(s) are credited and that the original publication in this journal is cited, in accordance with accepted academic practice. No use, distribution or reproduction is permitted which does not comply with these terms. 